Trixy G. Chu, MD'

Daniel Rafael R. Cachola III, MD²

Mary Agnes S. Regal, MD'

Agnes Cecille G. Llamas, MD'

Norberto V. Martinez, MD²

Wilfredo R. Santos, MD

'Department of Pediatrics

University of Santo Tomas Hospital

2Department of Otorhinolaryngology

Head and Neck Surgery

University of Santo Tomas Hospital
Correspondence: Dr. Daniel Rafael R. Cachola III

Department of Otorhinolaryngology

Head and Neck Surgery

University of Santo Tomas Hospital

España Blvd., Sampaloc, Manila 1015

Philippines

Phone: (632) 7313001 local 2411

Email: archangel_00011@yahoo.com

The authors declared that this represents original material that is not being considered for publication or has not been published or accepted for publication elsewhere, in full or in part, in print or electronic media; that the manuscript has been read and approved by all the authors, that the requirements for authorship have been met by each author, and that each author believes that the manuscript represents honest work.

Disclosures: The authors signed disclosures that there are no financial or other (including personal) relationships, intellectual passion, political or religious beliefs, and institutional affiliations that might lead to a conflict of interest.

This study was exhibited as a Poster Presentation during the 51st Annual Convention of the Philippine Pediatric Society, Manila, Philippines, April 6, 2014 and the 32nd Meeting of the European Society of Paediatric Infectious Diseases, Dublin, Ireland May 8, 2014.

Presented at the Philippine Society of Otolaryngology Head and Neck Surgery Analytical Research Contest. October 23, 2014. Unilab Bayanihan Center, Pasig City.

\section{Pneumococcal Conjugate Vaccine (Non-Typeable Haemophilus influenzae (NTHi) Protein D, Diphtheria or Tetanus Toxoid Conjugates) in Prevention of Acute Otitis Media in Children: A Cohort Study}

\begin{abstract}
Objective: To compare the incidence of acute otitis media among children aged 2 to 6 months old in Sampaloc, Manila who were previously given 3 doses of pneumococcal conjugate vaccine (Non-Typeable Haemophilus influenzae (NTHi) protein D, diphtheria or tetanus toxoid conjugates) and those who did not receive the vaccine over a period of one year.
\end{abstract}

Methods:

Design: Cohort Study

Setting: $\quad$ Primary Health Center in Sampaloc, Manila, Philippines

Participants: Medical records of well children aged 2 to 6 months were reviewed for inclusion. Participants were categorized into vaccinated and unvaccinated groups. Both groups underwent baseline history and physical examination including otoscopy and any signs and symptoms of active ear infection were noted. Subjects were followed up for a period of one year on a monthly basis for signs or symptoms of acute otitis media.

Results: A total of 176 subjects participated in the study. The overall incidence of AOM among participants was $5.11 \%$ (9 out of 176). An AOM incidence of $3.75 \%$ ( 3 out of 80 ) and $6.25 \%$ (6 out of 96) was found among the exposed and unexposed groups, respectively. Fisher's exact test (one-tailed) $p$ value $=.34$, relative risk (RR) $.6(95 \% \mathrm{Cl} 0.155,2.323)$.

Conclusion: The results of this study showed no difference in the development of AOM in the two groups. However, based on the relative risk, Pneumococcal conjugate vaccine is still beneficial in preventing $\mathrm{AOM}$ in children.

\section{Keywords: Pneumococcal Conjugate Vaccine; Acute Otitis Media}

Acute otitis media (AOM) is a common diagnosis among pediatric patients. In the Philippine General Hospital (PGH), acute otitis media constitutes $0.9 \%$ of Otorhinolaryngology Out-Patient Department consults. ${ }^{1}$ By three years of age, $75-80 \%$ of children will have had at least one episode, with the peak incidence occurring before the age of two years. ${ }^{2} \mathrm{AOM}$ is clinically defined as an inflammation of the middle ear with rapid onset of signs and symptoms of less than 3 weeks duration. ${ }^{1}$ The most common causative bacteria in AOM are Streptococcus pneumoniae, Haemophilus influenzae, and Moraxella catarrhalis, with Streptococcus pyogenes and Staphylococcus 


\section{ORIGINAL ARTICLES}

aureus also reported in some cases. Streptococcus pneumoniae can be isolated from up to $50 \%$ of AOM effusions and is the most common cause of complications. ${ }^{2}$ Since heptavalent pneumococcal conjugate vaccine (PCV-7) was incorporated into the routine immunization schedule in the United States by mid 2000 several studies have demonstrated a dramatic decrease in invasive pneumococcal disease. ${ }^{3}$ In March 2009, A pneumococcal vaccine containing 10 serotype-specific polysaccharides conjugated to Haemophilus influenzae protein D, tetanus toxoid, and diphtheria toxoid as the carrier proteins was developed (PHiDCV10) was licensed in the European Union (Synflorix, GlaxoSmithKline Vaccines, Rixensart, Belgium).

Vaccines have been used to prevent pneumococcal disease for more than 30 years. In the United States, seven pneumococcal serotypes cause approximately $80 \%$ of invasive disease and represent approximately $60 \%$ of middle-ear isolates in children younger than age 2 years. ${ }^{4}$ Antibacterial treatment has shown good activity against pneumococcal AOM, but with the recent advent of antibiotic-resistant pneumococcal strains there is an increasing risk for serious and fatal infections. Preventive immunization against pneumococcal disease, especially in individuals at risk, may be preferred to treatment of existing infections. ${ }^{4}$

We hypothesized $(\mathrm{H} 1)$ that a population vaccinated with Pneumococcal Conjugate Vaccine (Non-Typeable Haemophilus influenzae (NTHi) Protein D, Diphtheria or Tetanus Toxoid Conjugates) would have a lower incidence of Acute Otitis Media compared to an unvaccinated group of children. We conducted this study to compare the incidence of Acute Otitis Media among children aged 2 months to 6 months old in Sampaloc, Manila who were previously vaccinated with 3 doses of Pneumococcal conjugate vaccine (Non-Typeable Haemophilus influenzae (NTHi) protein D, diphtheria or tetanus toxoid conjugates) and those who were not vaccinated with the vaccine over a period of one year.

\section{Study Design: Cohort Study}

\section{METHODS}

Setting: Local Health Center in Earnshaw, Sampaloc, Manila consisting of 30 barangays with approximately 600-700 inhabitants per barangay, approximately one-fourth of whom were 0-71 months old. The area was bounded by A. Lacson, Fajardo, Valencia and Earnshaw Streets on the outskirts of the University of Santo Tomas Hospital.

\section{Subjects}

With Institutional Review and Ethics Committee approval, 188 children meeting inclusion criteria from the selected Local Health Center in Earnshaw, Sampaloc, Manila were considered for this study. The target sample size computed using OpenEpi Version 3.01 (Open Source Epidemiologic Statistics for Public Health, www.OpenEpi.com, updated 2013/04/06) was 188 using an alpha of 0.95 and power of $80 \%$. Inclusion criteria for the vaccinated and unvaccinated groups were well children born term (>37 weeks-42 weeks) who were or were not previously vaccinated with Pneumococcal Conjugate Vaccine (Non-Typeable Haemophilus influenzae (NTHi) Protein D, Diphtheria or Tetanus Toxoid Conjugates) between 2 to 6 months of age, respectively.

Excluded were children who were born preterm or post-term, children with weight $z$ scores of $<2$ or $>2$, length z scores of $<2$ or $>2$, weight for length of $<2$ or $>2$, children suffering from chronic illness (including chronic heart, lung, kidney, or liver disease; brain or spinal fluid leaks), those with weakened immune system (cancer, organ or bone marrow transplantation, children under chemotherapy or radiation treatment, long term steroids), suffering from sickle cell disease, with disorders of the spleen or those who underwent splenectomy.

\section{Procedure}

The investigators obtained permission to access medical records of the pediatric subjects aged 0-71 months from the local health officer. All available medical records were reviewed by an independent third party. Barangay health workers personally visited potential subjects in their homes and requested them to come to the local health center to be seen by the investigators. Study details were discussed with the parents or guardians of each child prior to study enlistment and written informed consent was obtained. Subjects who met the inclusion criteria and participated in the study were categorized by an independent third party to the vaccinated group and the unvaccinated group. Both groups underwent history and physical examination including otoscopy by the investigators at the initial visit to the local health center. Any sign and symptom of active ear infection was noted. The investigators were blinded to the vaccinated and unvaccinated groups. The diagnosis of acute otitis media was based on predefined clinical criteria, ${ }^{1}$ requiring 1) a history of acute onset of signs and symptoms, and 2) signs and symptoms of middle ear inflammation. Elements of the definition of acute otitis media were: 1) recent (within a time frame of less than three weeks), usually abrupt onset of signs and symptoms of middle ear inflammation; 2) any one of the following otoscopic findings: markedly retracted tympanic membrane, distinct erythema of the tympanic membrane, bulging of the tympanic membrane, limited or absent mobility of the tympanic membrane, air-fluid level or air bubbles behind the tympanic membrane, perforation with otorrhea; and 3) any one of the following: fever, distinct otalgia (discomfort clearly referable to the ears) that results in interference with or precludes normal activity or sleep). ${ }^{1}$ Participating subjects were required to be brought to the local health center and were actively monitored through clinical examination including otoscopy by the investigators for a period of one year on a monthly basis. The parents or guardians were also given a list of symptoms to record and were asked to bring their children to the study clinic for symptoms suggestive of acute otitis media. Subjects who were unable to come to the local health center were personally visited at home by the investigators.

\section{Statistical Analysis}

The overall incidence of acute otitis media as well as incidence of AOM among the vaccinated and unvaccinated groups was computed. The 
1-sided Fischer's exact test was computed using the Simple Interactive Statistical Analysis (SISA) Fischer Exact test calculator (Quantitative Skills Consultancy for Research and Statistics, The Netherlands) to compare the vaccinated and unvaccinated groups. The risk ratio was also computed using MedCalc (MedCalc Software, Belgium).

\section{RESULTS}

A total of 188 subjects were included in the study, aged between 2 months and 6 months at the time of enrolment. Of these, 176 participants completed the study while 12 participants were lost to follow-up. Out of the 176 participants, 80 were categorized to the vaccinated group and 96 were categorized to the unvaccinated group. The vaccinated group had 45 males and 35 females while the unvaccinated group had 42 males and 54 females. The mean age was 4.5 months in the vaccinated group and 5.7 months in the unvaccinated group.

The overall incidence of acute otitis media among all participants was $5.11 \%$ (9 out of 176), with incidences of 3.75\% (3 out of 80 ) and $6.25 \%$ (6 out of 96 ) among the vaccinated and unvaccinated groups, respectively. The one-sided Fisher's exact test $p$ value $=.34$. Relative risk (RR) was .6 (95\% Cl 0.155, 2.323).

\section{DISCUSSION}

After the introduction of PCV-7 in the US, Non-typeable H. influenza NTHi became the most common pathogen for a period of time, but an increase in non-PCV-7 S. pneumoniae was also noted. ${ }^{5}$ In a study by Parra et al., among bacterial etiology for AOM, 64\% of samples were culture positive for bacterial pathogens with $\mathrm{H}$. influenzae and $\mathrm{S}$. pneumoniae as the leading causes of bacterial AOM, detected in 34\% and $29 \%$ of AOM episodes, respectively. ${ }^{5}$ The most commonly isolated S. pneumoniae serotypes were 19A, 19F and 23F. All H. influenzae isolates were identified as non-typeable. ${ }^{5}$ Based on epidemiologic data from the Health Protection Agency (HPA), 35.9\% of AOM cases were assumed to have been attributable to S. pneumoniae and $32.3 \%$ to NTHi. PHiD-CV is a 10-valent conjugate vaccine that includes an additional 3 serotypes $(1,5$, and $7 F)$ and uses a carrier protein derived from nontypable $H$ influenzae (NTHi) for 8 of the 10 serotypes included. ${ }^{6}$ By virtue of using protein D from NTHi as a carrier protein, PHiD-CV may offer additional protection against NTHi, an important cause of $\mathrm{AOM}$ in children. ${ }^{6}$ In another study, a seven valent conjugate vaccine was found to be more immunogenic than the polysaccharide pneumococcal vaccines and was $80-100 \%$ effective against vaccine-type invasive disease and $50-60 \%$ effective against vaccine-type pneumococcal otitis media. ${ }^{3}$ Routine immunization with pneumococcal conjugate vaccines should substantially reduce the morbidity, mortality, and costs associated with pneumococcal disease in children. ${ }^{3}$

This study showed no statistically significant difference in the incidence of acute otitis media between the vaccinated and unvaccinated groups. Similar results were seen in a cohort study among Australian aboriginal children, wherein the introduction of pneumococcal vaccination was not associated with significant changes in prevalence or age of onset of different otitis media outcomes or the incidence of acute otitis media or tympanic membrane perforation.? In comparison to the study by Eskola et al., the vaccine reduced the number of episodes of acute otitis media from any cause by $6 \%$, culture- confirmed pneumococcal episodes by $34 \%$, and the number of episodes due to the serotypes contained in the vaccine by $57 \% .^{8}$ The number of episodes attributed to serotypes that were cross-reactive with those in the vaccine was reduced by $51 \%$, whereas the number of episodes due to all other serotypes increased by $33 \% .^{8}$ Based on the relative risk in this study, pneumococcal conjugate vaccination is still beneficial in preventing AOM in children, comparable to the previous study.

This study only focused on one subset of the population and may not be representative of the entire population. Moreover, this study did not account for risk factors in developing acute otitis media such as socioeconomic status, number of household members, overcrowding, and housing conditions. Because this study diagnosed AOM clinically and did not use other methods such as tympanostomy with culture and sensitivity; it was unable to identify organisms that may have caused the disease. The observation period was limited only to one year, and some subjects may have developed AOM after the conclusion of this study. We recommend a more diverse population with a larger sample size and a longer observation period be considered by future studies.

Although the results of this study showed no difference in the incidence of AOM among the two groups, based on the relative risk, Pneumococcal conjugate vaccine may still be beneficial in preventing AOM in children.

\section{REFERENCES}

1. Magiba-Caro R, Acuin J, Jose E, Chiong C, Villafuerte C, Pontejos A, Nolasco F. Clinical Practice Guidelines on Acute Otitis Media in Children. Philippine Society of Otolaryngology - Head and Neck Surgery, Inc. 2006

2. Grevers G. Challenges in reducing the burden of otitis media disease: An ENT perspective. Int JPediatr Otorhinolaryngol. 2010 Jun; 74(6):572-577. DOI: 10.1016/j.ijporl.2010.03.049. PubMed PMID: 20409595.

3. Poehling KA, Szilagyi PG, Grijalva CG, Martin SW, LaFleur B, Mitchel E, Griffin M, et al. Reduction of frequent otitis media and pressure-equalizing tube insertions in children after introduction of pneumococcal conjugate vaccine. Pediatrics. 2007 Apr; 119(4): 707-715. DOI:10.1542/ peds.2006-2138. PubMeD PMID: 17403841. Erratum in Pediatrics. 2007 June; 119(6): 1270. DOI:10.1542/peds.2007-1030.

4. O'Brien KL, Santosham M. Potential impact of conjugate pneumococcal vaccines on pediatric pneumococcal diseases. Am JEpidemiol. 2004 Apr 1; 159 (7): 634-644. DOI: 10.1093/aje/kwh082. PubMed PMID: 15033641.

5. Parra MM, Aguilar GM, Echaniz - Aviles G, Rionda RG, Estrada Mde L, Cervantes Y, Hausdorff WP et al. Bacterial etiology and serotypes of acute otitis media in Mexican children. Vaccine. 2011 Jul 26; 29(33): 5544-5549. DOI: 10.1016/j.vaccine.2011.04.128. PubMed PMID: 21596081.

6. De Wals P, Black S, Borrow R, Pearce D. Modeling the impact of a new vaccine on pneumococcal and nontypable Haemophilus influenzae diseases: a new simulation model. Clinical Therapeutics. 2009 Oct; 31(10): 2152-2169. DOI: 10.1016/j.clinthera.2009.10.014. PubMed PMID:19922887.

7. Mackenzie GA, Carapetis JR, Leach AJ, Morris PS. Pneumococcal vaccination and otitis media in Australian Aboriginal infants: comparison of two birth cohorts before and after introduction of vaccination. BMC Pediatr. 2009 Feb 19; 9:14. DOI: 10.1186/1471-2431-9-14. PubMed PMID: 19228431. PubMedCentral PMCID: PMC2656498

8. Eskola J, Kilpi T, Palmu A, Jokinen J, Haapakoski J, Herva E, Makela PH, et al. Efficacy of a pneumococcal conjugate vaccine against acute otitis media. N Engl J Med. 2001 Feb 8, 344(6):403-409. DOI: 10.1056/NEJM200102083440602. PubMed PMID: 11172176. 